\title{
The Main Parameters of SPB Stars on the Basis of IUE Spectra
}

\author{
E. Niemczura \\ Astronomical Institute of the Wroclaw University, ul. Kopernika 11, \\ Wroclaw, Poland
}

\begin{abstract}
We derive mean stellar parameters for 14 SPB stars observed during the IUE satellite mission, using an algorithmic procedure fitting theoretical flux distributions (Kurucz, 1996) to the IUE low-resolution spectra. We focus our attention on the metallicity parameter $[\mathrm{m} / \mathrm{H}]$. We found the mean value of $[\mathrm{m} / H]=-0.27 \pm 0.22$.
\end{abstract}

\section{Introduction}

Knowledge of abundances of the iron-group elements in B-type stars is important for a number of reasons. In particular, the spectral lines of these elements are the major source of opacity in hot stars driving pulsations in $\beta$ Cephei and SPB stars by the classical $\kappa$-mechanism (Dziembowski \& Pamyatnykh, 1993; Gautschy \& Saio, 1993). Recently, Fitzpatrick \& Massa (1999) demonstrated the usefulness of the low-resolution IUE observations in deriving the metal abundance parameter, $[\mathrm{m} / H]$, simultaneously with the effective temperature $\left(T_{\mathrm{eff}}\right)$, surface gravity $(g)$, angular diameter $(\theta)$ and interstellar reddening parameter $(E(B-V))$. The method was also tested by Niemczura et al. (2000) and applied to the standard stars listed in Code et al. (1976).

\section{Observations and their analysis}

We used IUE NEWSIPS observations of SPB stars obtained with the large aperture from both long- and short-wavelength cameras with high and low spectral resolutions. The observations expressed in the absolute units were supplemented by the optical spectrophotometric measurements taken from the literature. We use the Johnson $V$ magnitudes if no optical spectrophotometric data were available. The method of analysis is based on the least-squares optimization algorithm (Fitzpatrick \& Massa, 1999; Niemczura et al., 2000), which enables us to obtain various parameters involved with stellar spectra. The original method was modified in such a way that the stellar gravities were obtained from evolutionary models calculated by Townsend (2001). We found the relation $\log g=-12.4294+4.4628 \log T_{\text {eff }}-0.8208 \log L / L_{\odot}$ to be valid for main-sequence models with masses between 3.0 and $6.5 \mathrm{M}_{\odot}$. The standard deviation for this formula is equal to 0.01 dex. In order to calculate $\log L / L_{\odot}$, knowledge of the stellar parallaxes is necessary. We use HIPPARCOS parallaxes (ESA, 1997). During the best-fit procedure, the current luminosity has to be corrected for 
Table 1. The best-fit parameters for the analysed SPB stars.

\begin{tabular}{lcccccc}
\hline HD & $\log T_{\text {eff }}$ & $\log g$ & $E(B-V)$ & $\theta$ & {$[m / H]$} & $\log L / L_{\odot}$ \\
\hline 1976 & $4.201 \pm 0.004$ & 3.46 & $0.081 \pm 0.004$ & $0.162 \pm 0.007$ & -0.33 & $3.48 \pm 0.02$ \\
25558 & $4.216 \pm 0.007$ & 3.63 & $0.100 \pm 0.010$ & $0.172 \pm 0.013$ & -0.37 & $3.36 \pm 0.04$ \\
26326 & $4.174 \pm 0.004$ & 3.67 & $0.001 \pm 0.004$ & $0.181 \pm 0.020$ & -0.40 & $3.08 \pm 0.17$ \\
27396 & $4.198 \pm 0.008$ & 3.95 & $0.140 \pm 0.009$ & $0.226 \pm 0.015$ & -0.32 & $2.87 \pm 0.06$ \\
37151 & $4.123 \pm 0.006$ & 4.26 & $0.036 \pm 0.006$ & $0.081 \pm 0.030$ & -0.20 & $2.08 \pm 0.03$ \\
74195 & $4.213 \pm 0.005$ & 3.56 & $0.006 \pm 0.004$ & $0.387 \pm 0.023$ & -0.42 & $3.42 \pm 0.03$ \\
74560 & $4.229 \pm 0.004$ & 4.05 & $0.006 \pm 0.004$ & $0.207 \pm 0.009$ & -0.08 & $2.91 \pm 0.02$ \\
147394 & $4.176 \pm 0.003$ & 3.90 & $0.011 \pm 0.003$ & $0.360 \pm 0.006$ & -0.31 & $2.81 \pm 0.01$ \\
160762 & $4.264 \pm 0.004$ & 3.75 & $0.045 \pm 0.003$ & $0.321 \pm 0.011$ & -0.47 & $3.47 \pm 0.03$ \\
181558 & $4.209 \pm 0.006$ & 4.08 & $0.090 \pm 0.005$ & $0.116 \pm 0.027$ & -0.36 & $2.77 \pm 0.16$ \\
182255 & $4.180 \pm 0.003$ & 4.15 & $0.012 \pm 0.003$ & $0.196 \pm 0.011$ & -0.62 & $2.53 \pm 0.03$ \\
183133 & $4.240 \pm 0.013$ & 3.92 & $0.107 \pm 0.025$ & $0.086 \pm 0.042$ & -0.27 & $3.13 \pm 0.06$ \\
208057 & $4.225 \pm 0.003$ & 4.03 & $0.006 \pm 0.020$ & $0.190 \pm 0.008$ & -0.10 & $2.91 \pm 0.06$ \\
215573 & $4.152 \pm 0.002$ & 4.07 & $0.016 \pm 0.003$ & $0.193 \pm 0.006$ & -0.23 & $2.47 \pm 0.02$ \\
\hline
\end{tabular}

the Lutz-Kelker (1975) bias (Jerzykiewicz \& Molenda-Zakowicz, 2000). First, we search for the best-fit solutions with the fixed values of $[m / H]$. Then we fit the Lagrangian polynomials to the relation $\chi^{2}([\mathrm{~m} / H])$ to find metallicity corresponding to the minimum of $\chi^{2}$. Table 1 shows the best-fit parameters (with errors) for the analysed SPB stars.

The UV spectral region of main-sequence $B$ stars is very rich in spectral lines of iron-group elements and our results may provide an important clue to fix the metallicity of these stars. Some questions remain open. In particular, a verification of the metallicities using high-resolution spectra is needed. We therefore plan to study high-resolution IUE observations for known SPB stars in the near future.

\section{References}

Code, A.D., David, J., Bless, R.C., \& Hanbury Brown, R. 1976, ApJ, 203, 417 Dziembowski, W.A. \& Pamyatnykh, A.A. 1993, MNRAS, 262, 204

ESA, 1997, The Hipparcos and Tycho Catalogues

Fitzpatrick, E.L. \& Massa, D. 1999, ApJ, 525, 1011

Gautchy, A. \& Saio, H. 1993, MNRAS, 262, 213

Jerzykiewicz, M. \& Molenda-Zakowicz, J. 2000, AcA, 50, 369

Kurucz, R. 1996, CD-ROM, No. 13 and 19

Lutz, T.E. \& Kelker, D.H. 1973, PASP, 85, 573

Niemczura, E., Daszynska, J., \& Cugier, H. 2000, ASR, in press

Townsend, R.H.D. 2001, MNRAS, submitted 